\title{
A quantal Tolman temperature
}

\author{
Yongwan Gim ${ }^{\mathrm{a}}$, Wontae Kim ${ }^{\mathrm{b}}$ \\ Department of Physics, Sogang University, Seoul 121-742, South Korea
}

Received: 24 September 2015 / Accepted: 27 October 2015 / Published online: 24 November 2015

(C) The Author(s) 2015. This article is published with open access at Springerlink.com

\begin{abstract}
The conventional Tolman temperature based on the assumption of the traceless condition of energymomentum tensor for matter fields is infinite at the horizon if Hawking radiation is involved. However, we note that the temperature associated with Hawking radiation is of relevance to the trace anomaly, which means that the traceless condition should be released. So, a trace anomaly-induced Stefan-Boltzmann law is newly derived by employing the first law of thermodynamics and the property of the temperature independence of the trace anomaly. Then, the Tolman temperature is quantum-mechanically generalized according to the anomaly-induced Stefan-Boltzmann law. In an exactly soluble model, we show that the Tolman factor does not appear in the generalized Tolman temperature which is eventually finite everywhere, in particular, vanishing at the horizon. It turns out that the equivalence principle survives at the horizon with the help of the quantum principle, and some puzzles related to the Tolman temperature are also resolved.
\end{abstract}

\section{Introduction}

The proper temperature of the gravitating system of a perfect fluid in thermodynamic equilibrium has been defined by the well-known Tolman temperature [1,2]. In a static geometry, it assumes: (i) the perfect fluid of radiation in thermal equilibrium, (ii) the covariant conservation law of energymomentum tensor, (iii) the traceless condition of energymomentum tensor, (iv) the Stefan-Boltzmann law. The resulting temperature in the proper frame is written as

$T_{\mathrm{T}}=\frac{C}{\sqrt{-g_{00}(r)}}$,

where the Tolman factor appears in the denominator and $C$ is a constant determined by a boundary condition. For exam-

\footnotetext{
a e-mail: yongwan89@ sogang.ac.kr

be-mail: wtkim@sogang.ac.kr
}

ple, for the Schwarzschild black hole, the constant used to be determined by $C=T_{\mathrm{H}}$, where $T_{\mathrm{H}}$ is the Hawking temperature of the black hole $[3,4]$. As expected, the Tolman temperature becomes the Hawking temperature at infinity, whereas it is infinite at the horizon due to the blue-shifted Tolman factor which was discussed in Ref. [5]. It is worth noting that the Tolman temperature is for the freely falling observer at rest rather than the fixed observer who undergoes an acceleration [2]. For the fixed observer placed at the radius $r$ of the Schwarzschild black hole, the temperature can be expressed as the red/blue-shifted Hawking temperature

$T_{\mathrm{F}}=\frac{T_{H}}{\sqrt{-g_{00}(r)}}$,

where the red/blue-shift factor comes from the time dilation in the presence of the gravitational field at different places [6]. The fixed temperature is infinite at the horizon, which can also be understood in terms of the Unruh effect for the large black hole by keeping the detector in place [7], since the Unruh temperature is infinite at the horizon because of the infinite acceleration of the frame.

First, it would be interesting to note that the two temperatures (1) and (2) are the same in spite of the apparently different physical backgrounds; the former is for the inertial frame and the latter is for the fixed one. Second, the infinite Tolman temperature at the horizon is much more puzzling unless $C=0$. The firewall paradox was debated in evaporating black holes [8] and a similar prediction based on different assumptions was given in Ref. [9]. Note that this paradox can also be found even in the static black hole, since the Tolman temperature (1) tells us that the freely falling observer encounters quanta of the super-Planckian frequency at the horizon in the Hartle-Hawking-Israel state $[10,11]$. The recent work for the firewall issue in thermal equilibrium claims the existence of the massless firewall [12] whose energy density is negligible but temperature is infinite at the 
horizon. Eventually, it leads to the violation of the equivalence principle at the horizon.

On the other hand, it was shown that the equivalence principle can be restored at the horizon by invoking that at the horizon the Unruh temperature measured by the accelerating detector is the same as the temperature measured by the fixed detector in a gravitational field [13]. Additionally, in the Hartle-Hawking-Israel state, the energy density and pressure are finite at the horizon even though the Tolman temperature is infinite at the horizon $[14,15]$. It implies that the StefanBoltzmann law to relate the energy density (or the pressure) to the temperature must be nontrivial, which has been unsolved yet. Even worse, the energy density at the horizon is negative in the Hartle-Hawking-Israel state, so that it seems to be nontrivial task to relate the negative energy density to the positive temperature if the conventional Stefan-Boltzmann law is just assumed. In these respects, it raises some natural questions. In spite of the finite energy density at the horizon, what is the reason why the Tolman temperature is divergent at the horizon? So, is there any consistent Stefan-Boltzmann law to relate the energy density to the temperature covering the whole region?

In this work, we would like to investigate the Tolman temperature intensively in order to resolve the above issues in the regime of the standard quantum field theory and thermodynamics. To shed light on the essential feature of our formulation with exact solvability, we adopt the two-dimensional approach to the problems. First of all, we note that the energymomentum tensor of matter fields on the classical background metric receives semiclassical quantum corrections which give rise to the trace anomaly [16]. It means that the Tolman relation (1) is correct for the traceless case; however, it should be generalized semiclassically for a consistent formulation when Hawking radiation is involved, since Hawking radiation is indeed related to the trace anomaly of matter fields [17]. To get the consistent local proper temperature of the black hole, the conditions (iii) and (iv) among the four assumptions in the original Tolman's derivation should be released ab initio.

In Sect. 2, in the presence of the trace anomaly, we will derive a trace anomaly-induced Stefan-Boltzmann law by using the first law of thermodynamics and the nice property of the temperature independence of the trace anomaly [18], and naturally obtain the generalized Tolman temperature which can be reduced to the conventional Tolman temperature if the traceless condition is met.

In Sect. 3, for the exactly soluble two-dimensional Schwarzschild black hole, we shall show that the generalized Tolman temperature becomes finite everywhere and it vanishes at the horizon without the Tolman factor. As a result, it will be shown that the equivalence principle survives at the horizon thanks to the quantum principle, and the abovementioned questions in connection with the Tolman temper- ature are also resolved. Finally, conclusion and discussion will be given in Sect. 4 .

\section{Tolman temperature from trace anomaly-induced Stefan-Boltzmann law}

We start with a two-dimensional line element given as

$\mathrm{d} s^{2}=-f_{1}(r) \mathrm{d} t^{2}+f_{2}(r) \mathrm{d} r^{2}$,

where $f_{1}(r)$ and $f_{2}(r)$ are static functions and the metric is assumed to be asymptotically flat. In the static system, the overall macroscopic velocity of radiation flow is zero, and the velocity can be written as

$u^{\mu}=\frac{\mathrm{d} x^{\mu}}{\mathrm{d} \tau}=\left(\frac{1}{\sqrt{f_{1}(r)}}, 0\right)$.

The radiation is also regarded as a perfect fluid, so that the energy-momentum tensor is written as

$T^{\mu \nu}=(\rho+p) u^{\mu} u^{\nu}+p g^{\mu \nu}$,

where $\rho=T_{\mu \nu} u^{\mu} u^{\nu}$ and $p=T_{\mu \nu} n^{\mu} n^{\nu}$ are the local proper energy density and pressure, respectively, and $n^{\mu}$ is the spacelike unit normal vector satisfying $n^{\mu} n_{\mu}=1$ and $n^{\mu} u_{\mu}=0$. Note that the flux is also calculated as $\mathcal{F}=-T_{\mu \nu} u^{\mu} n^{\nu}$ which is zero in the static fluid corresponding to the thermal radiation in equilibrium $[10,11]$. Next, the covariant conservation law of the energy-momentum tensor can be written as $2 f_{1} \partial_{r} T_{r}^{r}=\left(T_{t}^{t}-T_{r}^{r}\right) \partial_{r} f_{1}$, which is reduced to

$2 f_{1} \partial_{r} p=-(\rho+p) \partial_{r} f_{1}$.

Next, the trace equation is given as

$-\rho+p=T_{\mu}^{\mu}$,

where the trace of the energy-momentum tensor is not always zero. Combining Eqs. (6) and (7), one can get

$\partial_{r}\left(f_{1} p\right)=\frac{1}{2} T_{\mu}^{\mu} \partial_{r} f_{1}$.

The resulting equation (8) is easily solved as

$p=\frac{1}{f_{1}}\left(C_{0}+\frac{1}{2} \int T_{\mu}^{\mu} \mathrm{d} f_{1}\right)$,

and

$\rho=\frac{1}{f_{1}}\left(C_{0}-f_{1} T_{\mu}^{\mu}+\frac{1}{2} \int T_{\mu}^{\mu} \mathrm{d} f_{1}\right)$, 
where the pressure and energy density are corrected by the trace anomaly, respectively. Note that the conventional Stefan-Boltzmann law in the two dimensional flat space is actually $p=\rho=\alpha T^{2}$ which is valid only in the absence of the trace anomaly, where $\alpha$ is the Stefan-Boltzmann constant. From Eqs. (9) and (10), the pressure and energy density are no longer symmetric. Moreover, there are many different expressions satisfying the anomaly relation (7). To relate the pressure (9) and energy density (10) to the temperature uniquely, we should find the Stefan-Boltzmann law which is compatible with the presence of the trace anomaly.

Now, for our purpose, the first law of thermodynamics is considered as

$\mathrm{d} U=T \mathrm{~d} S-p \mathrm{~d} V$,

where $U, T, S$, and $V$ are the thermodynamic internal energy, temperature, entropy, and volume in the proper frame, respectively, and $U=\int \rho \mathrm{d} V$. Thus, the first law is rewritten in the form of

$$
\left.\frac{\partial U}{\partial V}\right|_{T}=\left.T \frac{\partial S}{\partial V}\right|_{T}-p .
$$

Using the Maxwell relation of $\partial S /\left.\partial V\right|_{T}=\partial p /\left.\partial T\right|_{V}$, we get

$\rho=\left.T \frac{\partial p}{\partial T}\right|_{V}-p$.

Next, we are going to use the fact that the trace anomaly is independent of the temperature [18], so that from Eq. (7) we can obtain

$$
\left.\frac{\partial \rho}{\partial T}\right|_{V}=\left.\frac{\partial p}{\partial T}\right|_{V}
$$

where $\left.\partial_{T} T_{\mu}^{\mu}\right|_{V}=0$. Plugging Eqs. (7) and (14) into Eq. (13) in order to eliminate the pressure and its derivative with respect to the temperature with the fixed volume, one can get the first order differential equation for the energy density given as

$2 \rho=\left.T \frac{\partial \rho}{\partial T}\right|_{V}-T_{\mu}^{\mu}$.

Solving Eq. (15), the energy density and pressure can be obtained as

$\rho=\gamma T^{2}-\frac{1}{2} T_{\mu}^{\mu}$,

and

$p=\gamma T^{2}+\frac{1}{2} T_{\mu}^{\mu}$, where they are reduced to the conventional ones for the traceless case if the integration constant $\gamma$ is identified with the two-dimensional Stefan-Boltzmann constant, for example, $\gamma=\alpha=\pi / 6$ for the massless scalar field [17]. Hence, from Eqs. (16) and (17), the temperature can be written as

$T=\sqrt{\frac{1}{\alpha}\left(p-\frac{1}{2} T_{\mu}^{\mu}\right)}=\sqrt{\frac{1}{\alpha}\left(\rho+\frac{1}{2} T_{\mu}^{\mu}\right)}$.

Therefore, the resulting generalized Tolman temperature by using Eqs. (9) or (10) is obtained as

$T=\frac{1}{\sqrt{\alpha f_{1}}} \sqrt{C_{0}-\frac{f_{1}}{2} T_{\mu}^{\mu}+\frac{1}{2} \int T_{\mu}^{\mu} \mathrm{d} f_{1}}$,

where the temperature is independent of $f_{2}$. Indeed, there appeared nontrivial contributions to the temperature from the trace anomaly. Note that it is reduced to the conventional Tolman temperature if the energy-momentum tensor is traceless, so that $T=C / \sqrt{f_{1}(r)}$, where $C=\sqrt{C_{0} / \alpha}$. In the asymptotic infinity, the trace parts in Eq. (19) vanish, and the constant $C_{0}$ can be determined by the usual boundary condition.

\section{Application to two-dimensional Schwarzschild black hole}

Let us now study how the generalized Tolman temperature (19) actually works in the two-dimensional Schwarzschild black hole, where the metric is given as

$f(r)=f_{1}(r)=\frac{1}{f_{2}(r)}=1-\frac{2 M}{r}$,

where $M$ is the mass of black hole and the Newton constant is set to $G=1$. Now, using the explicit trace anomaly for the massless scalar field as $T_{\mu}^{\mu}=R /(24 \pi)$ [16,17], the proper temperature (19) can be calculated by imposing the boundary condition of $C_{0}=\alpha /(8 \pi M)^{2}$ which gives the Hawking temperature at infinity,

$T=\frac{1}{8 \pi M \sqrt{f(r)}} \sqrt{1-4\left(\frac{2 M}{r}\right)^{3}+3\left(\frac{2 M}{r}\right)^{4}}$.

The quantities in the square root in Eq. (21) can be factorized as 


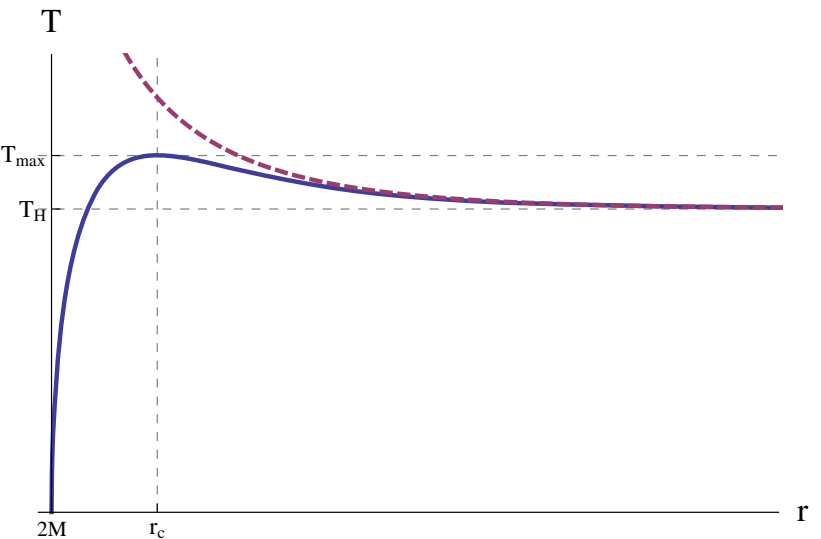

Fig. 1 The thick dotted curve is for the conventional Tolman temperature which is infinite at the horizon, whereas the solid curve is for the generalized one which is finite everywhere and especially goes to zero at the horizon. The maximum of the latter temperature $T_{\max }$ occurs at $r_{c} \sim 4 M$ in our model. The constant is set to $M=1$ for convenience. The infinite Tolman temperature at the horizon was suppressed by taking into account the trace anomaly

$$
\begin{aligned}
T & =\frac{1}{8 \pi M \sqrt{f(r)}} \\
& \times \sqrt{\left(1-\frac{2 M}{r}\right)\left(1+\frac{2 M}{r}+\left(\frac{2 M}{r}\right)^{2}-3\left(\frac{2 M}{r}\right)^{3}\right)},
\end{aligned}
$$

and consequently the generalized Tolman temperature is obtained as

$T=\frac{1}{8 \pi M} \sqrt{1+\frac{2 M}{r}+\left(\frac{2 M}{r}\right)^{2}-3\left(\frac{2 M}{r}\right)^{3}}$.

Note that the Tolman factor does not appear, which is compared to the form of the conventional Tolman temperature (1). One of the most interesting things to distinguish from the conventional behaviors of the Tolman temperature is that it is finite everywhere, and it also has a maximum value of the temperature at $r_{c} \sim 4 M$ as seen from Fig. 1. In particular, the temperature vanishes at the horizon. The suppression of the infinite Tolman temperature at the horizon by means of the quantum-mechanical trace anomaly is reminiscent of that of the infinite intensity at the high frequency in Rayleigh-Jeans law by the quantum correction.

As a matter of fact, in the large black hole, the metric (20) could be described by the Rindler metric for the near horizon limit, and the Unruh effect tells us that the temperature is given as $T_{\mathrm{U}}=a / 2 \pi$ in terms of the proper acceleration, where the acceleration of the fixed frame is $a=M /\left(r^{2} \sqrt{f(r)}\right)$ [7]. It implies that the free-fall observer would find the vanishing Unruh temperature, if the frame were free from the acceleration. So, it is reasonable for the observer in the proper frame to get the vanishing temperature at the horizon rather than the infinite temperature. In addition to this, authors in Ref. [13] also showed that the temperature (2) measured by the fixed observer in the gravitational background is generically higher than the Unruh temperature of the accelerating observer; however, they are the same at the event horizon of the black hole, so that the equivalence principle in the quantized theory is restored at the horizon. Thus, the vanishing generalized Tolman temperature at the horizon is compatible with the result that the equivalence principle is recovered at the horizon in Ref. [13].

Let us make a comment on the energy density and pressure. Plugging the generalized Tolman temperature (23) into Eqs. (16) and (17), one can obtain

$$
\begin{aligned}
& \rho=-\frac{1}{48 \pi r^{4} f(r)}\left(8 M r f(r)+2 M^{2}-\frac{r^{4}}{8 M^{2}}\right) \\
& p=\frac{1}{384 \pi M^{2}}\left[1+\frac{2 M}{r}+\left(\frac{2 M}{r}\right)^{2}+\left(\frac{2 M}{r}\right)^{3}\right]
\end{aligned}
$$

where the energy density and pressure near the horizon are negative and positive finite as $-\rho=p=1 /\left(96 \pi M^{2}\right)$, while $\rho=p=1 /\left(384 \pi M^{2}\right)=(\pi / 6) T_{H}^{2}$ at infinity. In a selfcontained manner, let us confirm whether the above energy density and pressure calculated by employing the generalized Tolman temperature are consistent with the results from direct calculations or not. For this purpose, in the light-cone coordinates defined as $\sigma^{ \pm}=t \pm r^{*}$ through $r^{*}=r+2 M \ln (r / M-2)$, the proper velocity (4) can be written as $u^{+}=u^{-}=1 / \sqrt{f(r)}$, where $u^{ \pm}=$ $u^{t} \pm u^{r} / f(r)$ and $n^{+}=-n^{-}=1 / \sqrt{f(r)}$. The components of the energy-momentum tensor are expressed as $T_{ \pm \pm}=-(1 / 48 \pi)\left(2 M f(r) / r^{3}+M^{2} / r^{4}\right)+(1 / 48) t_{ \pm}$and $T_{+-}=-(1 / 48 \pi)\left(2 M f(r) / r^{3}\right)$, where $t_{ \pm}$are the integration functions obtained from the integration of the covariant conservation law. The energy density and pressure measured in the freely falling frame can be calculated as $\rho=$ $-1 /\left(48 \pi r^{4} f(r)\right)\left[8 M r f(r)+2 M^{2}-\pi r^{4}\left(t_{+}+t_{-}\right)\right][19]$ and $p=1 /\left(48 \pi r^{4} f(r)\right)\left[-2 M^{2}+\pi r^{4}\left(t_{+}+t_{-}\right)\right]$, where we used the definition for the freely falling energy density and pressure. Since the radiation flow in the Hartle-Hawking-Israel state is characterized by choosing the integration functions as $t_{ \pm}=1 /\left(16 \pi M^{2}\right)$ [20], one can easily see that Eqs. (24) and (25) derived from the generalized Tolman temperature (23) are coincident with the above energy density and pressure based on the standard calculations.

\section{Discussion and conclusion}

It would be interesting to compare our computations with a previous result. The temperature (23) looks different 
from the free-fall temperature at rest, $T_{\mathrm{BT}}(r)=(1 / 8 \pi M)$ $\sqrt{1+2 M / r+(2 M / r)^{2}+(2 M / r)^{3}}$ [21] calculated by using the global embedding of the four-dimensional Schwarzschild black hole into a higher dimensional flat spacetime [22]. For example, the value of $T_{\mathrm{BT}}$ at the horizon is larger than that of the temperature at infinity, precisely, $T_{\mathrm{BT}}(2 M)=2 T_{\mathrm{H}}$ which is a maximum. Simply, we cannot conclude that the difference between them comes from the dimensionality, since we can exactly get the same free-fall temperature as $T_{\mathrm{BT}}$ for the two-dimensional Schwarzschild black hole (20) by using a slight different higher-dimensional embedding method [23]. Instead, we consider the new expression for the Stefan-Boltzmann law such as $p=\alpha T^{2}$ and $\rho=\alpha T^{2}-T_{\mu}^{\mu}$, then $T_{\mathrm{BT}}$ can be obtained from Eq. (25); however, this does not satisfy the relation (13) which comes from the first law of thermodynamics. Therefore, if the first law of thermodynamics is valid in the proper frame, the unique StefanBoltzmann law can be obtained thermodynamically among diverse expressions to satisfy the anomaly equation (7).

For the massless firewall in Ref. [12], it was claimed that it is massless but hot in the Hartle-Hawking-Israel state of black holes. At first sight, this phenomenon seems to be plausible in that the energy density and pressure at the horizon are at most negligible order of $1 / M^{2}$ in comparison with that of the temperature. Moreover, the infinite Tolman temperature at the horizon indicates the existence of the hot object. However, employing the generalized temperature (23), one could evade the infinite temperature at the horizon, and thus save the violation of the equivalence principle.

In conclusion, we have shown that the conventional Tolman temperature derived from the assumption of the traceless condition of energy-momentum tensor for matter fields was generalized, since the temperature associated with Hawking radiation is related to the trace anomaly. The most important ingredient in our formulation is that the Stefan-Boltzmann law was generalized in the presence of the trace anomaly by using the first law of thermodynamics and the property of the temperature independence of the trace anomaly. As a result, we obtained the generalized Tolman temperature which can be reduced to the conventional Tolman temperature if the traceless condition is met. In terms of the two-dimensional Schwarzschild black hole, we showed that the generalized Tolman temperature becomes finite everywhere and, in particular, it vanishes at the horizon, while it approaches the Hawking temperature at infinity. The quantum principle does not always give rise to conflicts but sometimes plays a key role to maintaining the equivalence principle. We hope that such a modification of the temperature as Eq. (19) will provide some clues about paradoxical problems in quantum gravity. Finally, it would be interesting to generalize this approach to higher dimensional black holes and other gravitational models, including some classical models whose traces are nontrivial.

Acknowledgments We would like to thank M. S. Eune and Edwin J. Son for exciting discussions. This work was supported by the National Research Foundation of Korea (NRF) Grant funded by the Korea government(MSIP) (2014R1A2A1A11049571).

Open Access This article is distributed under the terms of the Creative Commons Attribution 4.0 International License (http://creativecomm ons.org/licenses/by/4.0/), which permits unrestricted use, distribution, and reproduction in any medium, provided you give appropriate credit to the original author(s) and the source, provide a link to the Creative Commons license, and indicate if changes were made.

Funded by SCOAP ${ }^{3}$.

\section{References}

1. R.C. Tolman, Phys. Rev. 35, 904 (1930)

2. R.C. Tolman, P. Ehrenfest, Phys. Rev. 36(12), 1791 (1930)

3. S.W. Hawking, Nature 248, 30 (1974)

4. S.W. Hawking, Commun. Math. Phys. 43, 199 (1975) [Erratumibid. 46, 206 (1976)]

5. V.P. Frolov, A. Zelnikov, Introduction to Black Hole Physics (Oxford, New York, 2011)

6. S. Weinberg, Gravitation and cosmology (Wiley, New York, 1972)

7. W.G. Unruh, Phys. Rev. D 14, 870 (1976)

8. A. Almheiri, D. Marolf, J. Polchinski, J. Sully, JHEP 1302, 062 (2013). arXiv:1207.3123 [hep-th]

9. S.L. Braunstein, S. Pirandola, K. Zyczkowski, Phys. Rev. Lett. 110, $101301(2013)$

10. J.B. Hartle, S.W. Hawking, Phys. Rev. D 13, 2188 (1976)

11. W. Israel, Phys. Lett. A 57, 107 (1976)

12. W. Israel, A massless firewall. arXiv:1403.7470 [gr-qc]

13. D. Singleton, S. Wilburn, Phys. Rev. Lett. 107, 081102 (2011). arXiv:1102.5564 [gr-qc]

14. D.N. Page, Phys. Rev. D 25, 1499 (1982)

15. M. Visser, Phys. Rev. D 54, 5103 (1996). arXiv:gr-qc/9604007

16. S. Deser, M.J. Duff, C.J. Isham, Nucl. Phys. B 111, 45 (1976)

17. S.M. Christensen, S.A. Fulling, Phys. Rev. D 15, 2088 (1977)

18. H. Boschi-Filho, C.P. Natividade, Phys. Rev. D 46, 5458 (1992)

19. M. Eune, Y. Gim, W. Kim, Mod. Phys. Lett. A 29(40), 1450215 (2014). arXiv:1401.3501 [hep-th]

20. A. Wipf, Lect. Notes Phys. 514, 385 (1998). arXiv:hep-th/9801025

21. E.J. Brynjolfsson, L. Thorlacius, JHEP 0809, 066 (2008). arXiv:0805.1876 [hep-th]

22. S. Deser, O. Levin, Phys. Rev. D 59, 064004 (1999). arXiv:hep-th/9809159

23. R. Banerjee, B.R. Majhi, Phys. Lett. B 690, 83 (2010). arXiv:1002.0985 [gr-qc] 